\title{
Patient-prosthesis mismatch in patients treated with transcatheter aortic valve implantation - predictors, incidence and impact on clinical efficacy. A preliminary study
}

\author{
Karol Zbroński ${ }^{1, \star}$, Bartosz Rymuza ${ }^{1, \star}$, Piotr Scisło ${ }^{1}, K^{2}$ ajetan Grodecki ${ }^{1}$, Paulina Dobkowska ${ }^{1}$, Marek Wawrzacz $^{1}$, \\ Radosław Wilimski ${ }^{2}$, Anna Słowikowska ${ }^{2}$, Janusz Kochman ${ }^{1}$, Krzysztof J. Filipiak ${ }^{1}$, Grzegorz Opolski ${ }^{1}$, Zenon Huczek ${ }^{1}$
}

${ }^{1}$ First Chair and Department of Cardiology, Medical University of Warsaw, Warsaw, Poland

${ }^{2}$ Department of Cardiac Surgery, Medical University of Warsaw, Warsaw, Poland

${ }^{*}$ Authors equally contributed to the writing of the manuscript.

Adv Interv Cardiol 2017; 13, 4 (50): 281-287

DOI: https://doi.org/10.5114/aic.2017.71608

\begin{abstract}
A bstract
Introduction: Patient-prosthesis mismatch (PPM) is relatively frequent after surgical aortic valve replacement (SAVR) and negatively impacts prognosis.

Aim: We sought to determine the frequency and clinical effects of PPM after transcatheter aortic valve implantation (TAVI).

Material and methods: Overall, 238 patients who underwent TAVI were screened. Moderate PPM was defined as indexed effective orifice area (EOAi) between 0.65 and $0.85 \mathrm{~cm}^{2} / \mathrm{m}^{2}$, and severe PPM as $<0.65 \mathrm{~cm}^{2} / \mathrm{m}^{2}$. All-cause mortality and the Valve Academic Research Consortium 2 (VARC-2) defined composite of clinical efficacy at 1 year were the primary endpoints.

Results: Finally, 201 patients were included (mean age: $79.6 \pm 7.4$ years, 52\% females). The femoral artery served as the delivery route in $79 \%$ and most of the prostheses were self-expanding (68\%). Any PPM was present in 48 (24\%) subjects, and only 7 (3.5\%) had severe PPM. Body surface area (BSA) independently predicted any PPM (OR $=16.9, p<0.001)$ whereas post-dilation tended to protect against PPM $(\mathrm{OR}=0.46, p=0.09)$. All-cause mortality was similar in patients with moderate or severe PPM as compared to those with no PPM (14.6\% vs. $14.3 \%$ vs. $13.1 \%$, respectively, log-rank $p=0.98)$. Neither moderate $(\mathrm{OR}=1.6,95 \% \mathrm{Cl}: 0.8-3.2$, $p=0.16)$ nor severe PPM (OR $=1.67,95 \% \mathrm{Cl}: 0.36-7.7, p=0.51)$ had a significant impact on composite endpoint, or its elements, with the exception of transvalvular pressure gradient $>20 \mathrm{~mm} \mathrm{Hg}$.

Conclusions: Severe PPM after TAVI is rare, can be predicted by larger BSA and does not seem to affect mid-term mortality or composite clinical outcome. Larger studies are needed to find different independent predictors of PPM and elucidate its impact in terms of device durability and long-term clinical efficacy.
\end{abstract}

Key words: transcatheter aortic valve implantation, patient-prosthesis mismatch, effective orifice area.

\section{Introduction}

Transcatheter aortic valve implantation (TAVI) has become an alternative to surgical aortic valve replacement (SAVR) in intermediate and high-risk as well as inoperable patients with severe, symptomatic aortic stenosis. Patient-prosthesis mismatch (PPM) is a phenomenon in which the effective orifice area (EOA) of the otherwise functional valve is too small in relation to the patient. Incongruence is considered moderate in cases with aortic valve area indexed (AVAi) to body surface area less than $0.85 \mathrm{~cm}^{2} / \mathrm{m}^{2}$ and severe when below $0.65 \mathrm{~cm}^{2} / \mathrm{m}^{2}[1]$. Pa- tient-prosthesis mismatch in patients treated with SAVR has been shown to negatively impact functional class improvement and exercise tolerability and increase the adverse event rate as well as late mortality [2, 3].

The impact on survival and functional recovery of the same phenomenon in patients undergoing TAVI is not equally apparent.

\section{Aim}

We sought to further evaluate the predictors, the incidence and the importance for prognosis of PPM.

Corresponding author:

Piotr Scisło MD, PhD, First Department of Cardiology, Medical University of Warsaw, 1a Banacha St, 02-097 Warsaw, Poland, phone: +48 2259919 58, fax: +48 2259919 57, e-mail: scislo@wum.edu.pl

Received: 16.10 .2017 , accepted: 17.11.2017. 


\section{Material and methods}

\section{Study design and population}

Two hundred and thirty-eight consecutive inoperable or high-risk patients with severe symptomatic aortic stenosis (aortic valve area (AVA) $<1.0 \mathrm{~cm}^{2}$ or indexed valve area less than $0.6 \mathrm{~cm}^{2} / \mathrm{m}^{2}$ or mean gradient $>40 \mathrm{~mm} \mathrm{Hg}$ or maximum jet velocity $>4.0 \mathrm{~m} / \mathrm{s}$ or velocity ratio $<0.25)$ who after the heart team's decision underwent TAVI in a university heart center between March 2010 and March 2016 were screened. Both balloon- and self-expanding aortic valve prostheses of the first and second generation were used and all standard delivery routes were applied, with predominant use of transfemoral access. It was an all-comers study, and the only exclusion criterion was lack of a complete post-procedural echocardiographic study. Informed consent was obtained from all participating patients, and the local ethics committee granted permission for the study.

\section{Definitions, echocardiographic data}

Transthoracic echocardiography was performed at baseline and before discharge using the Philips Epiq 7C with $\times 5-1$ probe. Complete echocardiographic studies were performed in a standard fashion and analyzed by a single experienced echocardiographer. The left ventricular (LV) ejection fraction was calculated using the Simpson method. Low-gradient aortic stenosis was defined by a mean transaortic gradient of $<40 \mathrm{~mm} \mathrm{Hg}$ and an LV ejection fraction of < 35\% [4]. Before the procedure, AVA was estimated using the continuity equation approach (AVA $=$ LV outflow tract (LVOT) area $\times$ velocity time integral ${ }_{\text {LVOT }}$ /velocity time integral ${ }_{\text {valve }}$ ). After implantation, LVOT area was measured directly underneath the prosthesis, assuming a circular geometry. In order to correctly assess EOA, the pulse wave Doppler sample was located in the LVOT, adjacent to the in-flow segment of the prosthesis but not inside it, avoiding the region of subvalvular acceleration as previously described $[5,6]$. The indexed EOA was calculated by dividing the EOA by the patient's body surface area (BSA).

The PPM was defined as follows: no PPM if the indexed EOA was $>0.85 \mathrm{~cm}^{2} / \mathrm{m}^{2}$, moderate PPM if between 0.65 and $0.85 \mathrm{~cm}^{2} / \mathrm{m}^{2}$, and severe PPM when $<0.65 \mathrm{~cm}^{2} / \mathrm{m}^{2}$.

\section{Follow-up and endpoints}

Only patients discharged alive were included in the analysis. Clinical follow-up was performed either during a follow-up hospitalization or ambulatory visit 1, 6 and 12 months after the index hospitalization. Civil registries were additionally reviewed in order to assess patients' vital status.

The primary endpoint was all-cause mortality after discharge and up to 1 year follow-up, and the secondary endpoint was clinical efficacy after 30 days - a composite of all-cause mortality, all stroke, hospitalization for valve-related symptoms or worsening congestive heart failure, NYHA class III or IV or valve-related dysfunction (mean aortic valve gradient $\geq 20 \mathrm{~mm} \mathrm{Hg}$ or moderate or severe prosthetic aortic valve regurgitation) as proposed by Valve Academic Research Consortium-2 (VARC-2) [7]. Patient-prosthesis mismatch - part of the original endpoint - was removed from the composite.

\section{Statistical analysis}

Continuous variables, expressed as means \pm SD, were compared between the PPM and no PPM groups as well as between patients with and without an endpoint using Student's $t$-test or the Mann-Whitney U-test depending on the distribution pattern. The Shapiro-Wilk test was used to confirm or reject normal distribution of each continuous variable. Categorical variables, expressed as counts and percentages, were compared using the $\chi^{2}$ test or Fisher's exact test, as appropriate.

A univariate backwards likelihood ratio logistic regression model was used to identify predictors of the primary endpoint and its components.

Variables from the univariate analysis and baseline variables that were different between patients with and without PPM (with a $p$-value $\leq 0.10$ ) were included in the multivariate analysis. Results are presented as the odds ratio (OR) with 95\% confidence interval.

All probability values reported are 2 -sided and a value $<0.05$ was considered to be significant. All data were processed using SPSS software, version 22 (IBM SPSS Statistics, New York, US).

\section{Results}

\section{Population characteristics}

Of the 238 consecutive patients screened, complete post-procedural echocardiograms were either not available to the researchers or of suboptimal quality in 14 patients. Eighteen (7.6\%) patients were excluded due to peri-procedural or in-hospital non-valve-related death. Five patients in whom valve-in-valve TAVI for failed surgical prostheses was performed were also excluded. The overall study population consisted of $201 \mathrm{pa}-$ tients. The mean age was 79.6 years, there were 105 (52\%) females, over $75 \%$ of patients had hypertension, $40 \%$ had diabetes and almost half (47.3\%) were in New York Heart Association (NYHA) class III or IV. Detailed baseline characteristics of the study population are shown in Table I.

\section{Patient-prosthesis mismatch incidence and predictors}

Any PPM was present in 48 (23.9\%) subjects, 7 of whom had severe PPM (3.5\% of the group, $14.5 \%$ of the PPM cohort). Patients with any PPM had higher body 
Table I. Baseline, procedural and postprocedural characteristics of the entire cohort and within the PPM and no PPM groups

\begin{tabular}{|c|c|c|c|c|}
\hline Parameter & $\begin{array}{c}\text { Total } \\
N=201\end{array}$ & $\begin{array}{c}\text { Any PPM } \\
(n=48)(23.9 \%)\end{array}$ & $\begin{array}{c}\text { No PPM } \\
(n=153)(76.1 \%)\end{array}$ & $P$-value \\
\hline \multicolumn{5}{|l|}{ Baseline: } \\
\hline Age [years] & $79.63 \pm 7.4$ & $79.25 \pm 7.6$ & $79.75 \pm 7.4$ & 0.95 \\
\hline Female sex, $n(\%)$ & $105(52.2)$ & $27(56.3)$ & $78(51)$ & 0.52 \\
\hline Body surface area $\left[\mathrm{m}^{2}\right]$ & $1.81 \pm 0.18$ & $1.88 \pm 0.2$ & $1.79 \pm 0.2$ & 0.01 \\
\hline NYHA III/IV, $n(\%)$ & $95(47.3)$ & $23(47.9)$ & $72(47.1)$ & 0.92 \\
\hline Hypertension, $n(\%)$ & $151(75.5)$ & $34(72.3)$ & $117(76.5)$ & 0.57 \\
\hline Diabetes, $n(\%)$ & $78(39)$ & $18(38.3)$ & $60(39.2)$ & 0.91 \\
\hline COPD, $n(\%)$ & $37(18.4)$ & $11(22.9)$ & $26(17)$ & 0.36 \\
\hline Atrial fibrillation, $n(\%)$ & $70(35)$ & $18(38.3)$ & $52(34)$ & 0.59 \\
\hline History of MI, $n(\%)$ & $46(22.9)$ & $8(16.7)$ & $38(24.8)$ & 0.24 \\
\hline History of stroke, $n(\%)$ & $43(21.4)$ & $11(22.9)$ & $32(20.9)$ & 0.77 \\
\hline GFR $<30 \mathrm{ml} / \mathrm{min} / 1.73 \mathrm{~m}^{2}, n(\%)$ & $21(10.4)$ & $3(6.3)$ & $18(11.8)$ & 0.28 \\
\hline Ejection fraction (\%) & $51.1 \pm 13.9$ & $50.93 \pm 11.6$ & $51.14 \pm 14.5$ & 0.52 \\
\hline Bicuspid aortic valve, $n(\%)$ & $16(8)$ & $5(10.4)$ & $11(7.2)$ & 0.54 \\
\hline $\mathrm{AVA}\left[\mathrm{cm}^{2}\right]$ & $0.76 \pm 0.4$ & $0.72 \pm 0.2$ & $0.77 \pm 0.5$ & 0.95 \\
\hline AVAi $\left[\mathrm{cm}^{2} / \mathrm{m}^{2}\right]$ & $0.42 \pm 0.2$ & $0.38 \pm 0.9$ & $0.43 \pm 0.2$ & 0.5 \\
\hline $\mathrm{PGmax}[\mathrm{mm} \mathrm{Hg}]$ & $78.71 \pm 29.1$ & $82.31 \pm 33.9$ & $77.61 \pm 27.4$ & 0.73 \\
\hline PGmean [mm Hg] & $47.19 \pm 18.1$ & $48.36 \pm 19.7$ & $46.83 \pm 17.6$ & 0.9 \\
\hline \multicolumn{5}{|l|}{ Procedural, $n$ (\%): } \\
\hline Transfemoral access & $159(79.1)$ & $34(70.8)$ & $125(81.7)$ & 0.11 \\
\hline Predilatation & $141(70.5)$ & $37(77.1)$ & $104(68.4)$ & 0.28 \\
\hline Self-expanding valve & $137(68.2)$ & $32(66.7)$ & $105(68.6)$ & 0.8 \\
\hline Valve size $\leq 26 \mathrm{~mm}$ & $80(39.8)$ & $21(43.8)$ & $59(38.6)$ & 0.52 \\
\hline Postdilatation & $42(21)$ & $6(12.5)$ & $36(23.7)$ & 0.1 \\
\hline \multicolumn{5}{|l|}{ Postprocedural: } \\
\hline Ejection fraction (\%) & $54.78 \pm 12.2$ & $57.72 \pm 8.9$ & $53.9 \pm 12.9$ & 0.19 \\
\hline $\mathrm{EOA}\left[\mathrm{cm}^{2}\right]$ & $1.7 \pm 0.2$ & $1.44 \pm 0.2$ & $1.78 \pm 0.2$ & $<0.001$ \\
\hline EOAi $\left[\mathrm{cm}^{2} / \mathrm{m}^{2}\right]$ & $0.94 \pm 0.1$ & $0.77 \pm 0.1$ & $1 \pm 0.1$ & $<0.001$ \\
\hline PGmax [mm Hg] & $19.7 \pm 12.9$ & $24.69 \pm 13.4$ & $18.16 \pm 12.3$ & $<0.001$ \\
\hline PGmean [mm Hg] & $10.44 \pm 5.7$ & $14.35 \pm 8$ & $9.19 \pm 4$ & $<0.001$ \\
\hline
\end{tabular}

AVA - aortic valve area, AVAi-indexed aortic valve area, COPD - chronic obstructive pulmonary disease, EOA - effective orifice area, EOAi-indexed effective orifice area, GFR - glomerular filtration rate, NYHA - New York Heart Association, PG - pressure gradient.

surface area (BSA) and trended towards lower frequency of bioprosthesis post-dilation (Table I). In multivariate regression, BSA was found to be a highly independent predictor of PPM $(\mathrm{OR}=16.9, p<0.001)$ whereas postdilation tended to have a protective effect against PPM after TAVI (OR $=0.46, p=0.09)$ (Table II).

\section{Endpoints}

At 1 year 27 patients had died (13.4\%). Overall 95 (47.3\%) patients met at least 1 element of the composite clinical efficacy endpoint in the 1-year follow-up. There were $8(3.9 \%)$ strokes, 9 (4.4\%) patients were in NYHA class III or higher, 20 (9.9\%) had an unplanned hospital- 
Table II. Predictors of any PPM occurrence

\begin{tabular}{|c|c|c|c|}
\hline Parameter & Odds ratio (OR) & $95 \% \mathrm{Cl}$ & $P$-value \\
\hline Female sex & 1.24 & $0.64-2.37$ & 0.52 \\
\hline Body surface area $\left[\mathrm{m}^{2}\right]$ & 16.86 & $2.48-114.77$ & 0.004 \\
\hline Age [years] & 0.99 & $0.95-1.04$ & 0.69 \\
\hline Diabetes & 0.96 & $0.49-1.88$ & 0.91 \\
\hline Hypertension & 0.81 & $0.38-1.69$ & 0.57 \\
\hline Chronic obstructive pulmonary disease & 1.45 & $0.66-3.21$ & 0.36 \\
\hline Atrial fibrillation & 1.21 & $0.61-2.37$ & 0.59 \\
\hline Prior myocardial infarction & 0.61 & $0.26-1.41$ & 0.24 \\
\hline Prior stroke & 1.12 & $0.52-2.45$ & 0.77 \\
\hline Ejection fraction (\%) & 0.99 & $0.93-1.03$ & 0.94 \\
\hline Aortic valve area $\left[\mathrm{cm}^{2}\right]$ & 0.61 & $0.17-2.17$ & 0.45 \\
\hline Indexed aortic valve area $\left[\mathrm{cm}^{2} / \mathrm{m}^{2}\right]$ & 0.19 & $0.01-3.36$ & 0.26 \\
\hline Pressure gradient mean [mm Hg] & 1.01 & $0.99-1.02$ & 0.63 \\
\hline Pressure gradient max [mm Hg] & 1.01 & $0.99-1.02$ & 0.36 \\
\hline Annulus size [mm] & 0.95 & $0.85-1.05$ & 0.32 \\
\hline Bicuspid aortic valve & 1.5 & $0.49-4.56$ & 0.47 \\
\hline Prosthesis size $\leq 26 \mathrm{~mm}$ & 1.24 & $0.64-2.39$ & 0.52 \\
\hline Prosthesis size $\leq 23 \mathrm{~mm}$ & 1.21 & $0.37-3.97$ & 0.75 \\
\hline Self-expanding prosthesis & 0.91 & $0.46-1.82$ & 0.8 \\
\hline Predilatation & 1.55 & $0.73-3.3$ & 0.25 \\
\hline Postdilatation & 0.46 & $0.18-1.17$ & 0.09 \\
\hline
\end{tabular}

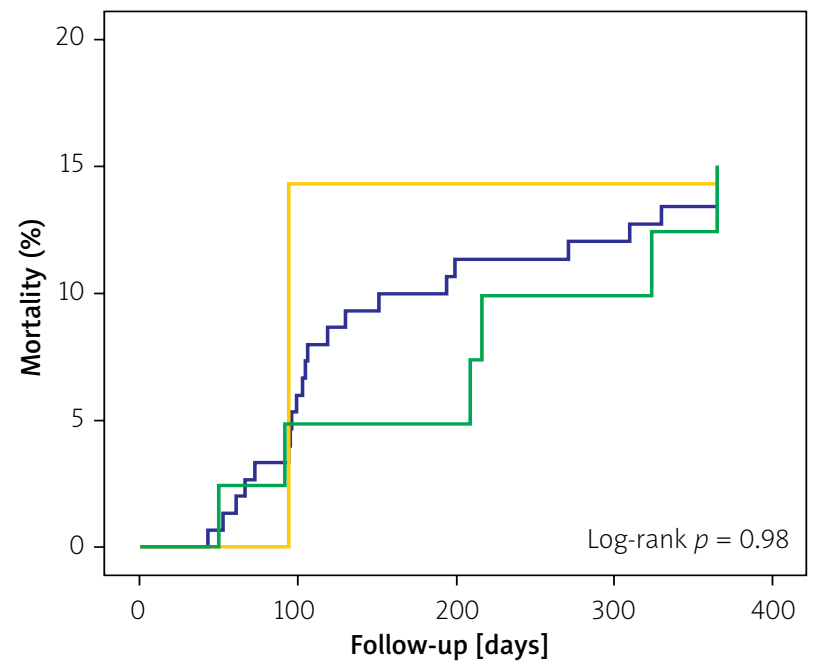

\begin{tabular}{|l|c|c|c|c|}
\hline Number at risk & Day 1 & Day 100 & Day 200 & Day 365 \\
\hline - No PPM & 153 & 144 & 136 & 133 \\
\hline - Moderate PPM & 41 & 39 & 39 & 35 \\
\hline - Severe PPM & 7 & 6 & 6 & 6 \\
\hline
\end{tabular}

Figure 1. Kaplan-Meier mortality curves according to the presence of PPM and its severity. Log-rank $p=0.92$ (severe PPM vs. no PPM) and log-rank $p=0.87$ (moderate PPM vs. no PPM) ization, 13 (6.5\%) had a mean pressure gradient across the prosthesis higher than $20 \mathrm{~mm} \mathrm{Hg}, 37$ (18.4\%) had moderate and 2 (0.9\%) severe aortic regurgitation.

\section{PPM and the primary endpoint}

There was no difference in the rates of all-cause mortality in patients with severe $(1 / 7,14.3 \%)$, moderate $(6 / 41,14.6 \%)$ or no PPM $(20 / 153,13.1 \%$; log-rank $p=0.98$, Figure 1). Similarly, presence of PPM did not impact the occurrence of composite endpoint of clinical efficacy at 1 year (Table III). When individual components were analyzed, both moderate and severe PPM significantly correlated with mean pressure gradient across the bioprosthesis greater than $20 \mathrm{~mm} \mathrm{Hg}$ (Table III). Finally, PPM also did not influence mortality at 1 year in patients without paravalvular regurgitation $(\mathrm{OR}=1.17,95 \% \mathrm{Cl}$ : $0.43-3.2, p=0.76$ ).

Analysis of indexed EOA as a continuous variable also demonstrated no discriminatory power in terms of predicting all-cause mortality (area under the curve (AUC) $0.53,95 \% \mathrm{Cl}: 0.42-0.65, p=0.58)$ or the composite endpoint of clinical efficacy (AUC $=0.52,95 \% \mathrm{Cl}$ : 0.44-0.6, $p=0.65$ ) (Figures 2 and 3). However, there was a clear discriminatory ability of EOAi in terms of predicting mean 
Table III. Impact of PPM on all-cause mortality, composite and individual endpoints of clinical efficacy

\begin{tabular}{|c|c|c|c|c|c|c|}
\hline \multirow[t]{2}{*}{ Parameter } & \multicolumn{3}{|c|}{ Moderate PPM $(n=41)(20.4 \%)$} & \multicolumn{3}{|c|}{ Severe PPM $(n=7)(3.5 \%)$} \\
\hline & OR & $95 \% \mathrm{Cl}$ & $P$-value & OR & $95 \% \mathrm{Cl}$ & $P$-value \\
\hline All-cause mortality & 1.14 & $0.43-3.05$ & 0.79 & 1.11 & $0.13-9.7$ & 0.93 \\
\hline Stroke & 1.26 & $0.24-6.47$ & 0.79 & - & - & - \\
\hline Unplanned hospitalization & 1.07 & $0.33-3.45$ & 0.91 & 3.97 & $0.7-22.4$ & 0.12 \\
\hline NYHA III/IV & 0.45 & $0.06-3.73$ & 0.46 & - & - & - \\
\hline PG mean $>20 \mathrm{~mm} \mathrm{Hg}$ & 10.5 & $1.96-56.2$ & 0.01 & 30.2 & $3.51-260$ & 0.002 \\
\hline AR moderate or severe & 1.15 & $0.5-2.67$ & 0.74 & 0.68 & $0.08-5.89$ & 0.73 \\
\hline Clinical efficacy (composite) & 1.6 & $0.8-3.2$ & 0.19 & 1.67 & $0.36-7.7$ & 0.51 \\
\hline
\end{tabular}

$A R$ - aortic regurgitation, NYHA - New York Heart Association, $P G$ - pressure gradient, PPM - patient-prosthesis mismatch.

pressure gradient $>20 \mathrm{~mm} \mathrm{Hg}$ after TAVI $(\mathrm{AUC}=0.79$, 95\% Cl: 0.62-0.96, $p=0.003$ ) (Figure 4).

\section{Discussion}

\section{Prevalence and predictors of PPM}

In our study, any PPM was found in less than a quarter of patients but severe PPM was diagnosed only in $3.5 \%$ of patients. Those numbers are well within the range of currently available reports for moderate PPM $[8,9]$, and on the lower side of the range for severe PPM $[6,8,10-15]$. These data may reassure that in TAVI with the predominant use of self-expanding prostheses and transfemoral access in most cases and anatomies a large enough EOAi can be achieved to avoid severe PPM. In the present work, we have not compared the frequency of PPM after TAVI and SAVR. However, a number of publications $[12,16,17]$ have reported that it is significantly less

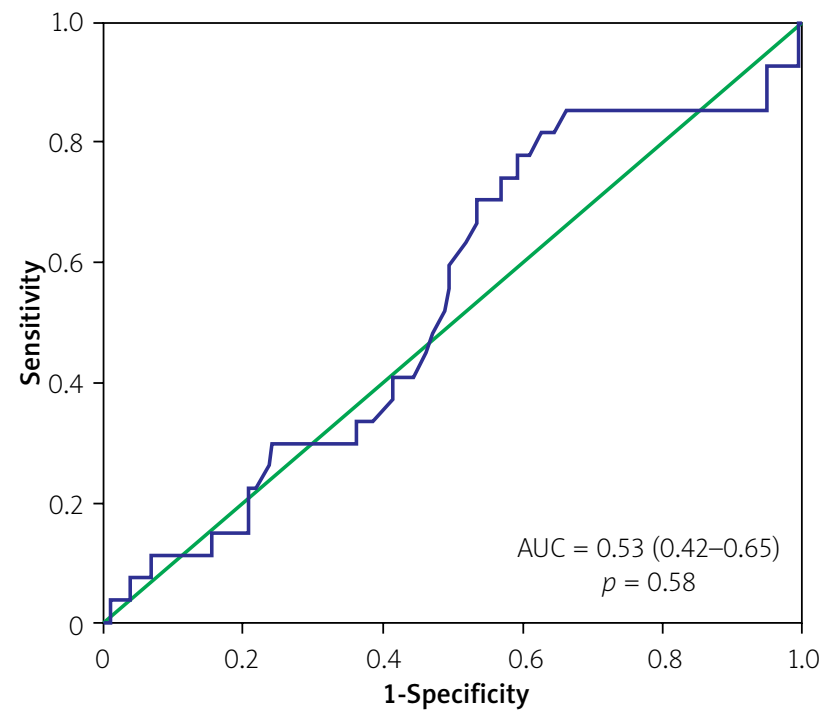

Figure 2. Receiver-operating characteristics curve for indexed EOA and all-cause mortality at 1 year after TAVI frequent after percutaneous treatment, with a recent meta-analysis showing that TAVI provides a $77 \%$ risk reduction of any mismatch as compared to SAVR [18].

In the analyzed population, the only predictor of PPM occurrence was BSA, a finding that is consistent with the available reports [9, 19-23]. A potential protective effect of bioprosthesis post-dilation on mismatch occurrence requires a larger sample size for confirmation. In this context, potentially adverse effects of post-dilation such as new bundle branch or atrioventricular block and stroke should be considered. We also cannot exclude that a similar percentage of PPM occurrence irrespective of the valve type used (self- vs. balloon-expanding) might also be potentially related to the limited sample size.

\section{Impact of PPM on mid-term prognosis}

In our study, there was no impact of either moderate or severe PPM on the occurrence of the clinical efficacy

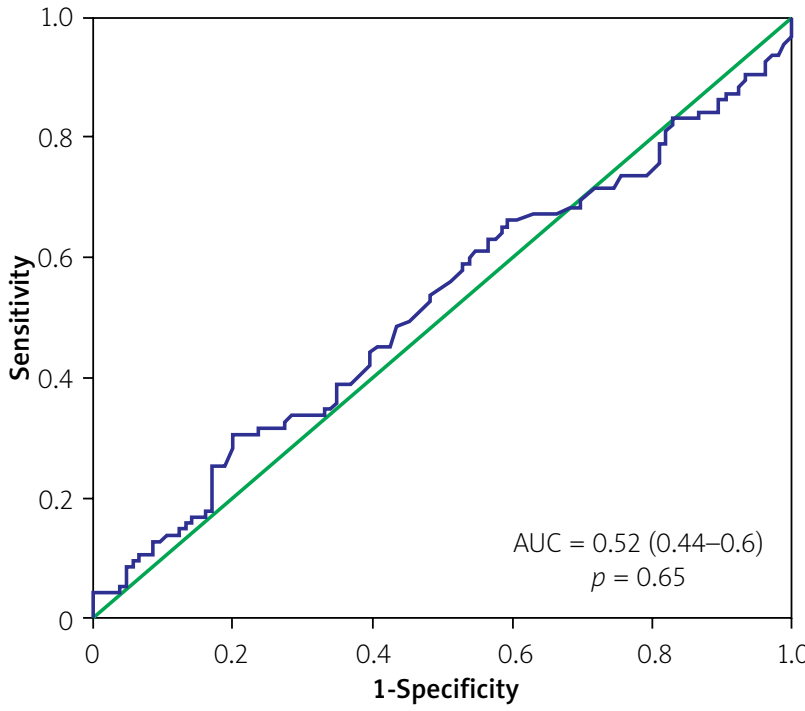

Figure 3. Receiver-operating characteristics curve for indexed EOA and composite clinical efficacy endpoint at 1 year after TAVI 


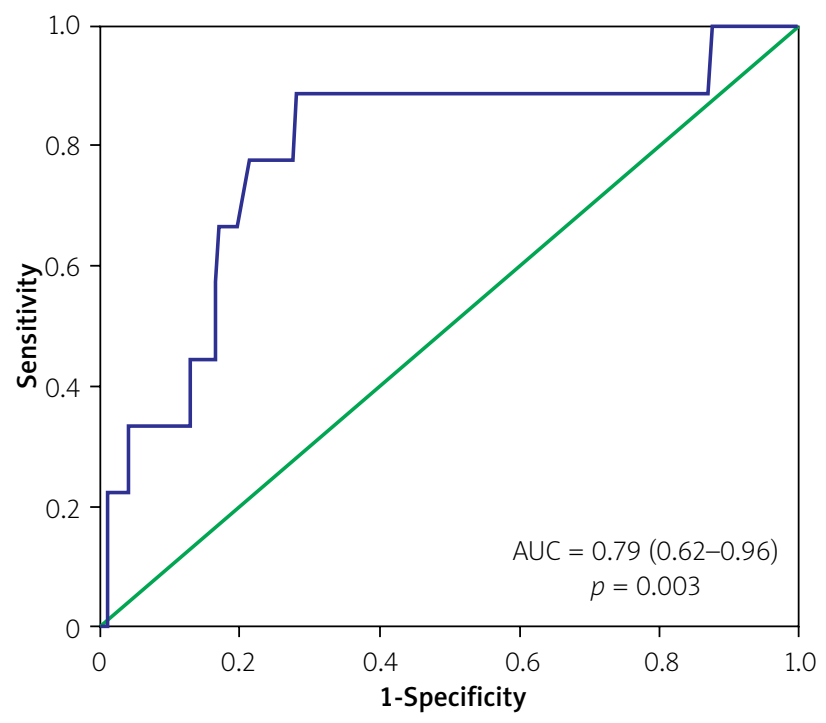

Figure 4. Receiver-operating characteristics curve for indexed EOA and transvalvular pressure gradient $>20 \mathrm{~mm} \mathrm{Hg}$ after prosthesis implantation

composite endpoint. Analysis of the endpoint components demonstrated a significant influence of mismatch on occurrence of high-pressure gradients (> $20 \mathrm{~mm} \mathrm{Hg}$ ) across the aortic valve after TAVI, which is consistent with previous studies $[9,19]$. Although a pressure difference is not part of the mismatch definition, its relation to a small effective orifice area is perfectly logical.

In regard to mortality, in the majority of available reports analyzing patients after TAVI no impact of PPM was found. In a recent meta-analysis [18], including 2654 patients, neither severe nor moderate mismatch influenced late mortality. Possible explanations of the lack of impact of PPM on survival in TAVI patients may include overestimation of mismatch in patients with a large BSA (e.g. obese subjects) along with the previously described "obesity paradox" [24] - better long-term outcome of patients with an elevated body mass index. Secondly, a confounding effect of coexisting moderate or severe paravalvular regurgitation is suggested. In the PARTNER trial cohort-A analysis [12] severe PPM worsened the survival only in patients without post-procedural AR. Our study did not corroborate these findings, with no impact of PPM irrespective of AR presence; nonetheless, the low number of patients with severe mismatch precludes definite conclusions.

\section{Limitations}

The main limitation of our study is the small sample size and single-center character. On the other hand, our study presents for the first time prevalence, predictors and impact on mortality of patient-prosthesis mismatch in a Polish population. Furthermore, apart from the PARTNER trial analysis and study by Kukucka et al., it is based on the largest subset of patients so far.

\section{Conclusions}

Severe PPM after TAVI is rare, can be predicted by larger baseline BSA and does not seem to affect midterm mortality or composite clinical outcome. Larger studies are needed to find different independent predictors of PPM and elucidate its impact in terms of device durability and long-term clinical efficacy.

\section{Conflict of interest}

Z.H. is a proctor for Medtronic. Other authors declare no conflict of interest.

\section{References}

1. Bax JJ, Delgado V, Bapat V, et al. Open issues in transcatheter aortic valve implantation. Part 2: procedural issues and outcomes after transcatheter aortic valve implantation. Eur Heart J 2014; 35: 2639-54.

2. Pibarot P, Dumesnil JG. Prosthesis-patient mismatch: definition, clinical impact, and prevention. Heart 2006; 92: 1022-9.

3. Mohty D, Malouf JF, Girard SE, et al. Impact of prosthesis-patient mismatch on long-term survival in patients with small St Jude Medical mechanical prostheses in the aortic position. Circulation 2006; 113: 420-6.

4. Monin JL, Monchi M, Kirsch ME, et al. Low-gradient aortic stenosis: impact of prosthesis-patient mismatch on survival. Eur Heart J 2007; 28: 2620-6.

5. Zoghbi WA, Chambers JB, Dumesnil JG, et al. Recommendations for evaluation of prosthetic valves with echocardiography and Doppler ultrasound: a report from the American Society of Echocardiography's Guidelines and Standards Committee and the Task Force on Prosthetic Valves, developed in conjunction with the American College of Cardiology Cardiovascular Imaging Committee, Cardiac Imaging Committee of the American Heart Association, the European Association of Echocardiography, a registered branch of the European Society of Cardiology, the Japanese Society of Echocardiography and the Canadian Society of Echocardiography, endorsed by the American College of Cardiology Foundation, American Heart Association, European Association of Echocardiography, a registered branch of the European Society of Cardiology, the Japanese Society of Echocardiography, and Canadian Society of Echocardiography. J Am Soc Echocardiogr 2009; 22: 975-1014.

6. Tzikas A, Piazza N, Geleijnse ML, et al. Prosthesis-patient mismatch after transcatheter aortic valve implantation with the medtronic CoreValve system in patients with aortic stenosis. Am J Cardiol 2010; 106: 255-60.

7. Kappetein AP, Head SJ, Généreux P, et al. Updated standardized endpoint definitions for transcatheter aortic valve implantation: the Valve Academic Research Consortium-2 consensus document (VARC-2). Eur J Cardiothorac Surg 2012; 42: S45-60.

8. Unbehaun A, Pasic M, Drews T, et al. New 29-mm balloon expandable prosthesis for transcatheter aortic valve implantation in large annuli. Ann Thorac Surg 2013; 95: 1982-90.

9. Bleiziffer S, Hettich I, Hutter A, et al. Incidence and impact of prosthesis-patient mismatch after transcatheter aortic valve implantation. J Heart Valve Dis 2013; 22: 309-16.

10. Sherif MA, Abdel-Wahab M, Awad O, et al. Early hemodynamic and neurohormonal response after transcatheter aortic valve implantation. Am Heart J 2010; 160: 862-9. 
11. Jilaihawi H, Chin D, Spyt T, et al. Prosthesis-patient mismatch after transcatheter aortic valve implantation with the Medtronic-Core valve bioprosthesis. Eur Heart J 2010; 31: 857-64.

12. Pibarot P, Weissman NJ, Stewart WJ, et al. Incidence and sequelae of prosthesis-patient mismatch in transcatheter versus surgical valve replacement in high-risk patients with severe aortic stenosis: a PARTNER trial cohort - a analysis. J Am Coll Cardiol 2014; 64: 1323-34.

13. Laflamme J, Puri R, Urena $M$, et al. Incidence and risk factors of hemolysis after transcatheter aortic valve implantation with a balloon-expandable valve. Am J Cardiol 2015; 115: 1574-9.

14. Munoz-García AJ, Munoz-García M, Carrasco-Chinchilla F, et al. Incidence and clinical outcome of prosthesis-patient mismatch after transcatheter aortic valve implantation with the CoreValve prosthesis. Int I Cardiol 2013; 167: 1074-6.

15. Spangenberg T, Budde U, Schewel D, et al. Treatment of acquired von Willebrand syndrome in aortic stenosis with transcatheter aortic valve replacement. JACC Cardiovasc Interv 2015; 8: 692700.

16. Finkelstein A, Schwartz AL, Uretzky G, et al. Hemodynamic performance and outcome of percutaneous versus surgical stentless bioprostheses for aortic stenosis with anticipated patient-prosthesis mismatch. J Thorac Cardiovasc Surg 2014; 147: 1892-9.

17. Kamperidis V, van Rosendael PJ, de Weger A, et al. Surgical sutureless and transcatheter aortic valves: hemodynamic performance and clinical outcomes in propensity scorematched highrisk populations with severe aortic stenosis. JACC Cardiovasc Interv 2015; 8: 670-7.

18. Takagi H, Umemoto T; ALICE (All-Literature Investigation of Cardiovascular Evidence) Group. Prosthesis-patient mismatch after transcatheter aortic valve implantation. Ann Thorac Surg 2016; 101: 872-80.

19. Ewe SH, Muratori M, Delgado V, et al. Hemodynamic and clinical impact of prosthesis-patient mismatch after transcatheter aortic valve implantation. J Am Coll Cardiol 2011; 58: 1910-8.

20. Kukucka M, Pasic M, Dreysse S, et al. Patient-prosthesis mismatch after transapical aortic valve implantation: incidence and impact on survival. J Thorac Cardiovasc Surg 2013; 145: 391-7.

21. Bagienski M, Kleczynski P, Dziewierz A, et al. Early- and mid-term outcomes after transcatheter aortic valve implantation. Data from a single-center registry. Adv Interv Cardiol 2016; 12: 122-7.

22. Bochenek T, Kusz B, Mizia M, et al. Echocardiographic evaluation of myocardial strain in patients after transcatheter aortic valve implantation. Postep Kardiol Inter 2015; 11: 95-9.

23. Stokłosa P, Szymański P, Dąbrowski M, et al. The impact of transcatheter aortic valve implantation on left ventricular performance and wall thickness - single-centre experience. Postep Kardiol Inter 2015; 11: 37-43.

24. Kodali SK, Williams MR, Smith CR, et al. Two-year outcomes after transcatheter or surgical aortic-valve replacement. N Engl J Med 2012; 366: 1686-95. 